\title{
Identification of a Shine-Through Artifact in the Trachea with ${ }^{124}$ I PET/CT
}

Sakar B. Abdul-Fatah, Mariangela Zamburlini, Servé G.E.A. Halders, Boudewijn Brans, Gerrit J.J. Teule, and Gerrit J. Kemerink

Department of Nuclear Medicine, Maastricht University Medical Center, Maastricht, The Netherlands

The presence of ${ }^{124}$ I in tissue near the trachea can cause a phenomenon that might be called shine-through. The effect is due to high-energy positrons that cross the air-filled trachea and annihilate at the opposite tracheal wall, incorrectly suggesting uptake at that location. Methods: We investigated shine-through using ${ }^{124} \mathrm{I},{ }^{68} \mathrm{Ga}$, and ${ }^{18} \mathrm{~F}$ PET/CT scans of a neck phantom. Additionally, we evaluated ${ }^{124}$ I studies of 29 patients with differentiated thyroid cancer who underwent imaging for postsurgical staging. Results: In the phantom studies with a $0.1-\mathrm{mL}^{124}$ I source, the relative intensity of shine-through decreased from $7 \%$ to nearly zero when the thickness of the positron-stopping layer was increased from 0.3 to $3.85 \mathrm{~mm}$. In patients, shine-through was observed in 5 of the 29 studies, with intensities between $0.7 \%$ and 14\%. Conclusion: Shine-through appears rather common in differentiated thyroid cancer. Recognition is important for identification of real lesions, calculation of uptake, and dosimetry.

Key Words: ${ }^{124}$ I PET/CT; thyroid disease; radioiodine; highenergy positrons

J Nucl Med 2009; 50:909-911

DOI: 10.2967/jnumed.108.060442

n the management of patients with differentiated thyroid cancer, ${ }^{124} \mathrm{I}$ PET/CT is becoming an indispensable tool because of its excellent diagnostic sensitivity $(1,2)$ and its use in PET-based dosimetry for planning ${ }^{131} \mathrm{I}$ therapy (3).

Especially with the postsurgical and preablative use of ${ }^{124} \mathrm{I}$, the high energy and the long range of the positrons can be a pitfall in image evaluation. The thyroid gland shares a common fascia with the trachea. Therefore, postsurgical remnants are usually fixed to the tracheal wall and rather close to the tracheal lumen. In this situation, a portion of the positrons may escape from the source area, cross the air-filled trachea, and annihilate at the opposite side of the trachea, thereby falsely suggesting activity at that location. Recognizing this shine-through is a prerequisite for correctly identifying lesions, quantifying ${ }^{124} \mathrm{I}$ uptake, and performing dosimetry. To assess this phenomenon quanti-

Received Nov. 20, 2008; revision accepted Mar. 9, 2009.

For correspondence or reprints contact: Gerrit J. Kemerink, Department of Nuclear Medicine, Maastricht University Medical Center,

P.O. Box 5800, 6202AZ Maastricht, The Netherlands.

E-mail: gerrit.kemerink@mumc.nl

COPYRIGHT (c) 2009 by the Society of Nuclear Medicine, Inc. tatively, we performed phantom experiments and analyzed the ${ }^{124}$ I patient data already available.

\section{MATERIALS AND METHODS}

\section{PET/CT Scans}

This study used a PET/CT scanner with a 64-slice CT component (Gemini TF; Philips). In all cases, low-dose CT (30 mAs) was performed. Using the standard Philips time-of-flight orderedsubsets expectation maximization reconstruction (33 subsets, 3 iterations), images corrected for random events, scattered radiation, and attenuation were obtained, as well as uncorrected images. In all patient studies, the matrix size was $144 \times 144(4 \times 4 \times 4 \mathrm{~mm}$ voxels), whereas the phantom studies were also reconstructed in a $288 \times 288$ matrix $(2 \times 2 \times 2 \mathrm{~mm}$ voxels $)$.

\section{Phantom Experiments}

In a neck phantom (acrylic, 140-mm diameter, 60-mm thickness), syringes of 1,2 , and $5 \mathrm{~mL}$ could be fitted in paratracheal holes near a $20-\mathrm{mm}$-diameter trachea. The volumes of liquid were $0.1,0.5$, and $1 \mathrm{~mL}$, respectively, containing between 0.3 and $1 \mathrm{MBq}$ of ${ }^{124} \mathrm{I}$. The minimum total thickness of plastic (stoppinglayer thickness) between the source and the tracheal cavity was $0.3,1.35,1.85,2.85,3.85$, and $4.85 \mathrm{~mm}$ for the $1-\mathrm{mL}$ syringe and $0.3,1.55$, and $2.55 \mathrm{~mm}$ for the $2-$ and $5-\mathrm{mL}$ syringes (Fig. 1). The phantom was scanned with one source at a time. Similar experiments were performed with ${ }^{18} \mathrm{~F}$ and ${ }^{68} \mathrm{Ga}$ using the $1-\mathrm{mL}$ syringe.

To simulate a thin remnant on the surface of the trachea, we impregnated with a solution of ${ }^{124} \mathrm{I}$ a disk of filter paper that was $12 \mathrm{~mm}$ in diameter and had a thickness of $8.8 \mathrm{mg} / \mathrm{cm}^{2}$. After the paper had dried, we glued it against the inner wall of the trachea.

On the images, we manually drew 2 volumes of interest, one encompassing all annihilations in the source area $\left(\mathrm{VOI}_{1}\right)$ and another encompassing all annihilations in the shine-through area $\left(\mathrm{VOI}_{2}\right)$. The shine-through percentage was calculated as $100 \% \times$ (annihilations in $\left.\mathrm{VOI}_{2}\right) /\left(\right.$ combined annihilations in $\mathrm{VOI}_{1}$ and $\mathrm{VOI}_{2}$ ).

\section{Analysis of Patient Data}

PET/CT had been performed on 29 patients with differentiated thyroid cancer at about $3 \mathrm{wk}$ after thyroidectomy and $1 \mathrm{wk}$ before radioiodine ablation. The scans were obtained at 24 and $96 \mathrm{~h}$ after oral administration of $20-25 \mathrm{MBq}$ of ${ }^{124} \mathrm{I}$.

Lesions that showed ${ }^{124}$ I uptake and were near the trachea were identified. Subsequently, the opposite tracheal regions were investigated for areas with annihilations. When such an area was found in both transverse and coronal cross sections, it was considered as due to shine-through only if its shape followed 
FIGURE 1. Schematic drawing of phantom source-trachea configuration in transverse plane and illustration of shinethrough as function of stopping-layer thickness for 1-mL syringe. Images from left to right are of stopping-layer thickness of $0.3,1.85,2.85$, and $3.85 \mathrm{~mm}$. Top row shows transverse slices; bottom row, coronal slices. Position of trachea, indicated by circle and straight lines, was taken from CT images. Upper-level window settings for images were, from left to right, $1.2 \%, 0.54 \%, 0.39 \%$, and $0.36 \%$ of image maximum.

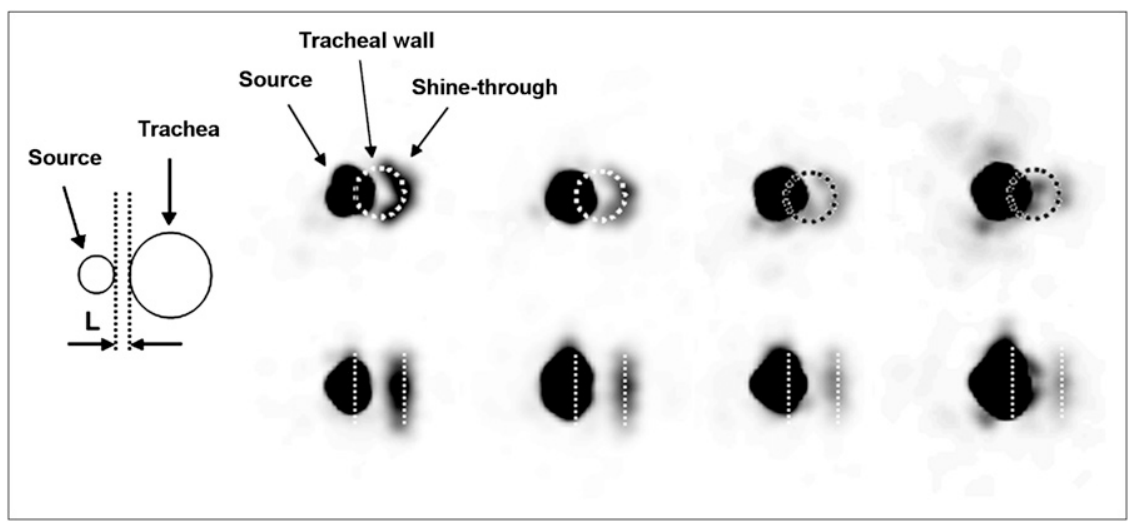

the tracheal wall, its centerline coincided approximately with the border of the trachea, and its intensity was above spurious background and noise.

Shine-through was computed as in the phantom study.

\section{RESULTS}

\section{Phantom Experiments}

Figure 1 shows the PET images obtained for the 1-mL syringe when the stopping-layer thickness was varied between 0.3 and $3.85 \mathrm{~mm}$. Figure 2 graphically presents the magnitude of the shine-through for all 3 syringes and for the various thicknesses of the stopping layer. The shinethrough for the filter paper source on the surface of the trachea was approximately $38 \%$.

Experiments with ${ }^{68} \mathrm{Ga}$ yielded results that were nearly identical to those for ${ }^{124} \mathrm{I}$ (e.g., for the $0.1-\mathrm{mL}$ source and 0.3 -mm layer, shine-through was $7.8 \%$ vs. $7.4 \%$ ), whereas for ${ }^{18} \mathrm{~F}$ the shine-through $(0.9 \%)$ was visible only for a stopping-layer thickness of $0.3 \mathrm{~mm}$.

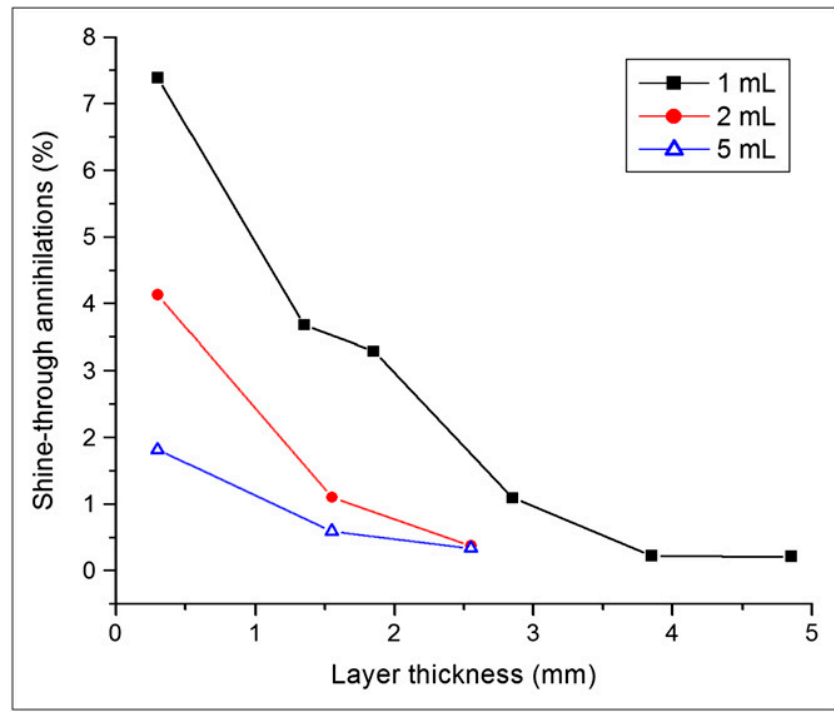

FIGURE 2. Relative magnitude of shine-through in phantom study for different source volumes as function of stopping-layer thickness.
The relative shine-through was quantitatively similar in corrected and uncorrected images.

\section{Patient Data}

The shine-through phenomenon was seen in 5 of the 29 patients (17\%), both at $24 \mathrm{~h}$ and at $96 \mathrm{~h}$. Figure 3 shows the shine-through in 4 patients at $96 \mathrm{~h}$ in transaxial and coronal cross-sections. Figure 4 shows similar data for the fifth patient, but at 24 and $96 \mathrm{~h}$. Table 1 presents the uptake in the ${ }^{124}$ I parent lesions as a percentage of the administered activity, as well as the relative magnitude of shine-through at 24 and $96 \mathrm{~h}$.

\section{DISCUSSION}

Our results show that the occurrence of shine-through depends on the positron range and the distance of the decaying nucleus from the trachea. The $\beta$-range is approximately $0.5 \cdot \mathrm{E}_{\beta, \max } / \rho \mathrm{cm}$, with $\mathrm{E}_{\beta, \max }$ being the maximum $\beta$-energy in $\mathrm{MeV}$, and $\rho$ the tissue density in $\mathrm{g} / \mathrm{cm}^{3}$. Therefore, in the case of ${ }^{124} \mathrm{I}$, where $2 \beta$-branches with $\mathrm{E}_{\beta, \max }$ values of 2.138 and $1.535 \mathrm{MeV}$ are dominant, the soft-tissue range is about $1 \mathrm{~cm}$. The intensity, however, drops steeply with distance (4), as can also be seen in Figure 2.

In this study, we calculated the shine-through from the ratio of the annihilations in the artifact to all annihilations
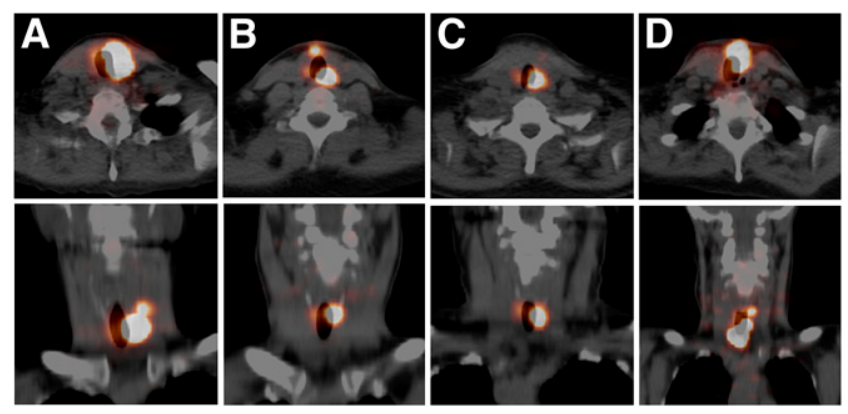

FIGURE 3. Shine-through in 4 patient studies at $96 \mathrm{~h}$ after oral intake of $20-25 \mathrm{MBq}$ of ${ }^{124}$. Top row shows transaxial PET/CT images; bottom row, coronal images. A-D correspond to patient codes in Table 1. Top level of viewing window was $29 \%, 4 \%, 24 \%$, and $2 \%$ of image maximum. 


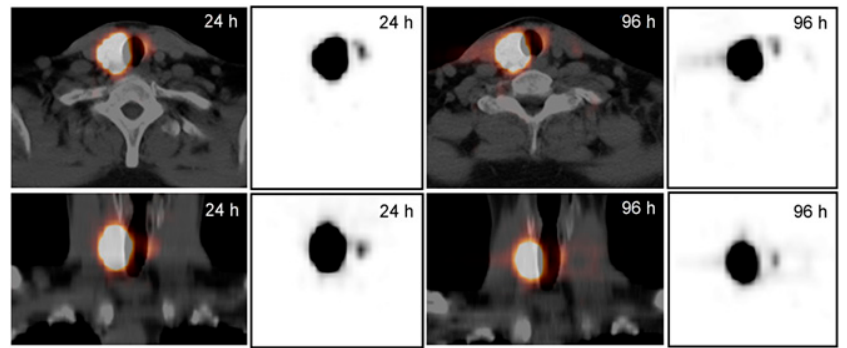

FIGURE 4. Shine-through in fifth patient (patient E) at 24 and $96 \mathrm{~h}$ after oral intake of ${ }^{124}$. Top row shows transaxial PET/CT images; bottom row, coronal images. Gray-scale PET-only images are shown for comparison with those of phantom in Figure 1.

associated with the parent source. This causes a dependence of shine-through on the form and the size of the activity distribution, with shine-through increasing in general with decreasing source volume (Fig. 2, effect of syringe size for fixed stopping-layer thickness). For a thin remnant in the tracheal wall, the ratio of shine-through might even be quite high, as was illustrated by the thin source we placed on the surface of the phantom trachea, yielding about $38 \%$ shine-through.

When the shine-through intensity falls below $1 \%$, it becomes difficult to distinguish it from spurious background. Some background is always present in PET, but the additional $\gamma$-radiation of the nonideal PET nuclide ${ }^{124} \mathrm{I}$ (5) might cause an increase.

The ${ }^{68} \mathrm{Ga}$ and ${ }^{18} \mathrm{~F}$ experiments aimed at excluding other potential explanations for the shine-through phenomenon. ${ }^{68} \mathrm{Ga}$ has an $\mathrm{E}_{\beta, \max }$ of $1.885 \mathrm{MeV}$, comparable to that of ${ }^{124} \mathrm{I}$, and the similarity between the results for ${ }^{68} \mathrm{Ga}$ and ${ }^{124} \mathrm{I}$ rules out the possibility that the $\gamma$-emissions in the decay of ${ }^{124} \mathrm{I}$ are a major cause of shine-through. Furthermore, the virtual absence of shine-through in ${ }^{18} \mathrm{~F}$ data excludes the possibility that scanner imperfections (e.g., in reconstruction) are causing the artifact. At the same time, the ${ }^{18} \mathrm{~F}$ results corroborate the conclusion that positron range effects are responsible, as ${ }^{18} \mathrm{~F}$ has a small $\mathrm{E}_{\beta, \max }$ of 0.634 $\mathrm{MeV}$. The similarity of shine-through in corrected and noncorrected images demonstrates, furthermore, that the effect is not caused by imperfections in the corrections, such as those due to misregistration between PET and CT.

Finally, it should be realized that shine-through can occur with any positron emitter that accumulates close enough to an air-filled cavity. Theoretically, this effect might happen at all air-containing spaces, for example,
TABLE 1. Relative Magnitude of Lesion Uptake and

Shine-Through in ${ }^{124}$ I PET Patient Studies

\begin{tabular}{cccc}
$\begin{array}{c}\text { Patient } \\
\text { code }\end{array}$ & $\begin{array}{c}\text { Lesion } \\
\text { uptake at } \\
24 \mathrm{~h} \mathrm{( \% )}\end{array}$ & $\begin{array}{c}\text { Shine-through } \\
\text { at } 24 \mathrm{~h}(\%)\end{array}$ & $\begin{array}{c}\text { Shine-through } \\
\text { at } 96 \mathrm{~h}(\%)\end{array}$ \\
A & 0.10 & 14.0 & 9.2 \\
B & 2.53 & 1.9 & 1.9 \\
C & 0.19 & 6.7 & 7.4 \\
D & 3.27 & 0.8 & 0.9 \\
E & 0.30 & 0.7 & 0.7 \\
\hline
\end{tabular}

within the respiratory system and the gastrointestinal tract. Another manifestation of positron escape is the "illumination" of surfaces surrounding thin-walled sources suspended in air.

\section{CONCLUSION}

Activity of ${ }^{124}$ I near the trachea can result in annihilations in the opposite wall of the trachea, incorrectly suggesting activity at that location. Phantom experiments showed that the effect decreases sharply with the thickness of the material between the activity and air cavity.

In $17 \%$ of the 29 patients who underwent ${ }^{124}$ I PET/CT after thyroidectomy, shine-through was observed, with a relative intensity between $0.7 \%$ and $14 \%$. The apparent activity in shine-through should be included in the activity of the source lesion to obtain the correct activity distribution.

\section{ACKNOWLEDGMENTS}

We thank Christian Urbach and Shahriar Tahmasebi for their help with preliminary experiments.

\section{REFERENCES}

1. Freudenberg LS, Antoch G, Frilling A, et al. Combined metabolic and morphologic imaging in thyroid carcinoma patients with elevated serum thyroglobulin and negative cervical ultrasonography: role of ${ }^{124} \mathrm{I}-\mathrm{PET} / \mathrm{CT}$ and FDG-PET. Eur J Nucl Med Mol Imaging. 2008;35:950-957.

2. Phan HT, Jager PL, Paans AM, et al. The diagnostic value of ${ }^{124}$ I-PET in patients with differentiated thyroid cancer. Eur J Nucl Med Mol Imaging. 2008;35:958965.

3. Jentzen W, Freudenberg L, Eising EG, Sonnenschein W, Knust J, Bockisch A. Optimized ${ }^{124}$ I PET dosimetry protocol for radioiodine therapy of differentiated thyroid cancer. J Nucl Med. 2008;49:1017-1023.

4. Blanco A. Positron range effects on the spatial resolution of RPC-PET. IEEE Nucl Sci Symp Conf Rec. 2006;4:2570-2573.

5. Jentzen W, Weise R, Kupferschläger J, et al. Iodine-124 PET dosimetry in differentiated thyroid cancer: recovery coefficient in 2D and 3D modes for PET/ (CT) systems. Eur J Nucl Med Mol Imaging. 2008;35:611-623. 